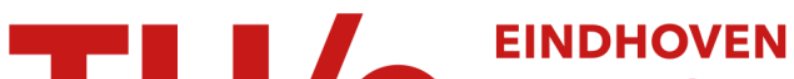 \\ UNIVERSITY OF \\ TECHNOLOGY
}

\section{On gradient-enhanced damage and plasticity models for failure in quasi-brittle and frictional materials}

\section{Citation for published version (APA):}

Borst, de, R., Pamin, J., Peerlings, R. H. J., \& Sluys, L. J. (1995). On gradient-enhanced damage and plasticity models for failure in quasi-brittle and frictional materials. Computational Mechanics, 17(1-2), 130-141.

https://doi.org/10.1007/s004660050098

DOI:

10.1007/s004660050098

Document status and date:

Published: 01/01/1995

\section{Document Version:}

Publisher's PDF, also known as Version of Record (includes final page, issue and volume numbers)

\section{Please check the document version of this publication:}

- A submitted manuscript is the version of the article upon submission and before peer-review. There can be important differences between the submitted version and the official published version of record. People interested in the research are advised to contact the author for the final version of the publication, or visit the $\mathrm{DOI}$ to the publisher's website.

- The final author version and the galley proof are versions of the publication after peer review.

- The final published version features the final layout of the paper including the volume, issue and page numbers.

Link to publication

\section{General rights}

Copyright and moral rights for the publications made accessible in the public portal are retained by the authors and/or other copyright owners and it is a condition of accessing publications that users recognise and abide by the legal requirements associated with these rights.

- Users may download and print one copy of any publication from the public portal for the purpose of private study or research.

- You may not further distribute the material or use it for any profit-making activity or commercial gain

- You may freely distribute the URL identifying the publication in the public portal.

If the publication is distributed under the terms of Article 25fa of the Dutch Copyright Act, indicated by the "Taverne" license above, please follow below link for the End User Agreement:

www.tue.nl/taverne

Take down policy

If you believe that this document breaches copyright please contact us at:

openaccess@tue.nl

providing details and we will investigate your claim. 


\title{
On gradient-enhanced damage and plasticity models for failure in quasi-brittle and frictional materials
}

\author{
R. de Borst, J. Pamin, R. H. J. Peerlings, L. J. Sluys
}

Abstract Gradient-enhanced damage and plasticity approaches are reviewed with regard to their ability to model focalization phenomena in quasi-brittle and frictional materials. Emphasis is put on the algorithmic aspects. For the purpose of carrying out large-scale finite element simulations efficient numerical treatments are outlined for gradient-enhanced damage and gradient-enhanced plasticity models. For the latter class of models a full dispersion analysis is presented at the end of the paper. In this analysis the fundamental role of dispersion in setting the width of localization bands is highlighted.

\section{1}

\section{Introduction}

Failure in cohesive-frictional materials involve localization of deformation, i.e. we observe that at incipient failure small zones of highly strained material develop rather abruptly, while the remainder of the structure experiences almost no additional straining. Examples are cracks in concrete, shear bands in soils and metals, dilatational bands in polymers and rock faults. Experiments show that these localization phenomena are accompanied by a more or less sharp decrease of the load-carrying capacity. This phenomenon is commonly named strain softening and can lead to ill-posed boundary value problems in standard continuum theories, since in quasi-static problems ellipticity of the governing set of differential equations is no longer assured, while in dynamic problems hyperbolicity can be lost locally. In numerical simulations we observe an extreme mesh sensitivity in terms of fineness and direction of the grid lines. To remedy this improper behaviour the standard

Communicated by S. N. Aturi, 18 August 1995

R. de Borst

Delft University of Technology, Faculty of Civil Engineering, Eindhoven University of Technology, Faculty of Mechanical Engineering, P.O. Box 3048, 2600 GA Delft, The Netherlands

J. Pamin

Cracow University of Technology, Cracow, Poland

R. H. J. Peerlings

Eindhoven University of Technology, Faculty of Mechanical

Engineering, Eindhoven, The Netherlands

L. J. Sluys

Delft University of Technology, Faculty of Civil Engineering, Delft,

The Nelherlands

Correspondence to: R. de Borst

Dedicated to J. C. Simo continuum model must be enriched. Here, yarious strategies are possible, such as nonlocal approaches (Pijaudier-Cabot and Bazant 1987, Bazant and Pijaudier-Cabot 1988, Simo 1988), micro-polar continua (Mühlhaus and Vardoulakis 1987, Mihlhaus 1989, de Borst 1991, 1993), rate-dependent approaches (Needleman 1988, Loret and Prévost 1990, Sluys and de Borst 1992, Sluys 1992), and continuum models enhanced with higher-order deformation gradients (Aifantis 1984, 1987, 1992, Coleman and Hodgdon 1985, Schreyer and Chen 1986, Lasry and Belytschko 1988, Vardoulakis and Aifantis 1991, Mühlhaus and Aifantis 1991, de Borst and Mühlhaus 1992, Pamin 1994).

This article is an updated version of a keynote lecture presented at the Fourth International Conference on Computational Plasticity (de Borst, Pamin and Sluys 1995), erriched with elements of a contributed lecture at the same conference (Peerlings, de Borst, Brekelmans and de Vree 1995a). We shall concentrate on the implications of using gradient-enhanced continuum models in large-scale numerical simulations of localization and failure. In particular, we shall consider gradient plasticity and gradient damage models, and we shall demonstrate how effective numerical strategies for these enhanced continua can be developed.

The article is laid out as follows. First, the general setting of the boundary value problem is given. Two classes of constitutive models are considered, namely gradient-enhanced isotropic damage and gradient-enhanced plasticity models. Then, the algorithms are developed for both classes of material models. It seems that the algorithm for the damage model is simpler than that for the gradient plasticity model, which observation is related to the issue of the additional boundary conditions that emerges in such higher-order continuum theories. Finally, a treatment is given of wave propagation in a gradient-enhanced plastic medium. The pivotal role of dispersion in setting the band width in enriched continua is emphasized.

\section{2}

\section{Enhanced continua}

\section{1}

\section{General framework}

For the formulation of the incremental boundary value problem in gradient-enhanced continua we introduce the displacement vector $\mathbf{u}=\left(u_{x}, u_{y}, w_{z}\right)$, the strain tensor in a vector form $\varepsilon=\left\{\hat{c}_{\mathrm{xx}}\right.$, $\left.\varepsilon_{y z}, \varepsilon_{z z}, \gamma_{x y}, \gamma_{y z}, \gamma_{z x}\right)$ and the stress tensor in a vector form $\sigma=\left(\sigma_{x=x}, \sigma_{y y}, \sigma_{z z z}, \sigma_{x x}, \sigma_{y z}, \sigma_{z x}\right)$. Setting $\mathbf{R}=\operatorname{diag}[\rho, \rho, \rho]$ with $\rho$ the mass density, we have, under the assumption of small deformations, the equations of motion for a body occupying 
a volume $V$

$\mathbf{L}^{T} \boldsymbol{\sigma}+\mathbf{b}=\mathbf{R} \mathbf{i}$

the kinematic equations

$\varepsilon=\mathrm{Lu}$,

and the constitutive equations, either in a total format

$\boldsymbol{\sigma}=\mathbf{f}(\varepsilon, \sigma, \mathfrak{q})$

or in a rate format

$\dot{\sigma}=g(\varepsilon, \sigma, q) \dot{\varepsilon}$

where a finite number of scalar or tensor-valued internal variables are conveniently collected in a vector $q$. In the above equations the superscript $T$ is the transpose symbol and a superimposed dot signifies differentiation with respect to time, so that a superimposed double dot implies that a quantity is differentiated twice with respect to time. $\mathbf{b}$ is a body-force vector and $\mathrm{L}$ is a differential operator matrix:

$\mathrm{L}^{T}=\left[\begin{array}{cccccc}\frac{\partial \cdot \partial}{\partial x} & 0 & 0 & \frac{\partial \cdot}{\partial y} & 0 & \frac{\partial \cdot}{\partial z} \\ 0 & \frac{\partial}{\partial y} & 0 & \frac{\partial \cdot}{\partial x} & \frac{\partial \cdot}{\partial z} & 0 \\ 0 & 0 & \frac{\partial}{\partial z} & 0 & \frac{\partial \cdot}{\partial y} & \frac{\partial \cdot}{\partial x}\end{array}\right]$.

To complete the incremental boundary value problem we specify the standard static and kinematic boundary conditions on complementary parts of the body surface $S$ :

$\Sigma v_{s}=\mathbf{t}, \quad \mathbf{u}=\mathbf{u}_{s}$

with $\Sigma$ the stress tensor in a matrix form, $v_{s}$ the outward normal to the surface of the body $S$ and $t$ the boundary traction vector, and the appropriate initial conditions in case of dynamic loadings. The additional boundary conditions that emerge in higher-order continuum models will be discussed next, when the specific constitutive formulations for damage and plasticity are treated.

\section{2}

\section{Gradient damage}

Herein, we shall restrict ourselves to isotropic damage formulations. In a strain-based formulation in the sense of Sino and $\mathrm{Ju}(1987)$ we then have

$\sigma=(1-\omega) \mathrm{D}^{e} \varepsilon$

with $D^{e}$ the virgin elastic stiffness matrix, and $\omega$ a scalar-valued internal variable, which reflects the amount of damage which the material has experienced. It starts at zero (undamaged

state) and grows to one (complete loss of integrity) as a function of a scalar history parameter $\pi$, which represents the most severe deformation the material has experienced: $\omega=\omega(x)$ and initiates at a threshold level $\kappa_{0}$. Damage growth is possible if the damage loading function

$f(\tilde{\varepsilon}, \pi)=\tilde{\varepsilon}-\kappa$

vanishes. In particular, the damage loading function $f$ and the rate of damage growth $\dot{\omega}$ have to satisfy the Kuhn-Tucker conditions

$f \leqq 0, \quad \dot{\omega} \geqq 0, \quad f \dot{\omega}=0$

In (7) $\tilde{\varepsilon}$ is the local equivalent strain, which can be a function of strain invariants, the principal strains, or the local energy release due to damage (Lemaitre and Chaboche 1990). In a non-local generalization the equivalent strain $\tilde{\varepsilon}$ is replaced by a spatially averaged quantity (Pijaudier-Cabot and Bazant 1987, Bazant and Pijaudier-Cabot 1988):

$\bar{\varepsilon}(\mathrm{x})=\frac{1}{\Omega_{r}(\mathrm{x})} \int_{\Omega} g(\mathbf{s}) \tilde{\varepsilon}(\mathbf{x}+\mathrm{s}) \mathrm{d} \Omega, \quad \Omega_{r}(\mathbf{x})=\int_{\Omega} g(\mathrm{~s}) \mathrm{d} \Omega$

with $g$ (s)a weight function, e.g, the error function, and $s$ a relative position vector pointing to the infinitesimal volume $\mathrm{d} \Omega$.

Non-local constitutive relations can be approximated by gradient models by expanding the kernel ô of the integral in ( 9 ) into a Taylor series (Mühlhaus and Aifantis 1991). Jf we truncate after the second-order terms and carry out the integration implied in (9) under the assumption of isotropy, the following relation ensues:

$\bar{\delta}=\bar{\varepsilon}+\bar{c} \nabla^{2} \tilde{E}$,

where $\bar{c}$ is a material parameter of the dimension length squared. It can be related to the averaging volume and then becomes dependent on the precise form of the weight function $g$. In here, we adopt a phenomenological view in that $\sqrt{c}$ is supposed to reflect the length scale of the failure process we wish to describe macroscopically.

Formulation (10) has a severe disadvantage when applied in a finite element context, namely that it requires computation of second-order gradients of the local equivalent strain $\tilde{\varepsilon}$. Since this quantity is a function of the strain tensor, and since the strain tensor involves first-order derivatives of the displacements, third-order derivatives of the displacements have to be computed, which inevitably necessitates $C^{1}$-continuity of the shape functions. To obviate this problem, Eq. (10) is differentiated twice and the result is substituted in Eq. (10). Again neglecting fourth-order terms this leads to

$\bar{\varepsilon}-\bar{c} \nabla^{2} \bar{\varepsilon}=\tilde{z}$

When $\bar{\varepsilon}$ is discretized independently and making use of the divergence theorem, a $C^{0}$-interpolation for $\vec{c}$ then suffices (Peerlings, de Borst, Brekelmans and de Vree 1995a, 1995b).

As stipulated before, higher-order continua require additional boundary conditions. With Eq. (11) governing the damage process, either the averaged strain $\bar{\varepsilon}$ itself or its normal derivative must be specified at the boundary of the body $S$ :

$\bar{\varepsilon}=\bar{\varepsilon}_{s}, \quad(\nabla \bar{\varepsilon})^{T} v_{s}=\bar{\varepsilon}_{n,}$, 
with the subscript $s$ denoting the boundary of the body $S$ (cf. Eq. (5)). The physical interpretation of these boundary conditions remains an open issue, but in the example catculations the natural boundary condition $\left(\bar{\varepsilon}_{n s}=0\right)$ has been adopted.

\section{3}

\section{Gradient plasticity}

The flow theory of plasticity adopted below departs from the strain-based damage model introduced in the preceding section essentially in the sense that the total relation of $\mathrm{Eq} .(3 \mathrm{a})$ is replaced by a rate formulation in the sense of $\mathrm{Eq} .(3 \mathrm{~b})$ :

$\dot{\boldsymbol{\sigma}}=\mathrm{D}^{e}(\dot{\varepsilon}-\dot{\lambda} \mathrm{m})$

with $\mathbf{m}$ the plastic flow direction and $\dot{\lambda}$ the flow intensity. Quite similar to damage theory, a loading function is introduced, say $f$, which in a stress-space plasticity theory as considered here, bounds the allowable stress states. The salient departure from standard plasticity theory is the dependence of the yield function on gradients of the equivalent plastic strain measure $\kappa$, in addition to the stresses $\sigma$ and the equivalent plastic strain $\kappa$ itself. In particular, we shall consider a yield function that depends on $\sigma, \kappa$ and the Laplacian of $\kappa$ (Mühlhaus and Aifantis 1991):

$f=f\left(\sigma, \kappa, \nabla^{2} \kappa\right)$

Analogously to damage theory the yield function $f$ and the rate of plastic staining $i$ have to satisfy the Kuhn-Tucker conditions

$\dot{\lambda} \geq 0, f \leqq 0, \quad f \dot{\lambda}=0$.

Henceforth we shail consider the case of a linear gradient dependence, so that the yield function can be written as:

$f=\phi(\sigma, \kappa)+\bar{c} \nabla^{2} \kappa$

with $\bar{c}$ again an additional material parameter which reflects the intensity of the gradient influence. As for the gradient damage theory it has the dimension of length squared and for vanishing values the gradient influence gradually disappears.

In general, the hardening parameter $\kappa$ is integrated along the loading path and its rate can be a function of invariants of the plastic strain rate, $t^{p}=m$, or the rate of plastic work. Herein, we limit our discussion to the strain-hardening hypothesis, so that

$\dot{\kappa}=\sqrt{\frac{2}{3}\left(\dot{\varepsilon}^{p}\right)^{T} \mathrm{Z} \dot{\varepsilon}^{p}}$

with $\mathrm{Z}=\operatorname{diag}\left[1,1,1, \frac{1}{2}, \frac{1}{2}, \frac{1}{2}\right]$. For nost commonly employed flow rules, definition (17) reduces to

$\dot{k}=n \dot{\lambda}$

with $\eta$ a positive constant, which depends on the adopted flow rule (Pamin 1994).
3

Algorithmic treatment of gradient damage

3.1

Formulation

For the development of the finite element formulation of the gradient-enhanced damage model we start by transforming the governing equations for motion (1) and the 'non-local' equivalent strain $\bar{\xi}$ Eq. (11), into a weak form:

$\int_{V} \delta \mathbf{u}^{T}\left(\mathbf{L}^{\top} \boldsymbol{\sigma}+\mathbf{b}-\mathbf{R} \mathbf{i}\right) \mathrm{d} V=0$,

$\int_{V} \delta \bar{\varepsilon}\left(\bar{\varepsilon}-\bar{c} \nabla^{2} \bar{\varepsilon}-\tilde{\varepsilon}\right) \mathrm{d} V=0$

where the $\delta$-symbol denotes the variation of a quantity. Using the divergence theorem, the kinematic relation (2), the boundary conditions (5) and (12), and the constitutive relation (6) we obtain

$\int_{V} \delta \mathbf{u}^{T} \mathbf{R u ̈} \mathrm{d} V+\int_{V}(1-\omega) \delta \varepsilon^{T} \mathbf{D}^{\varepsilon} \varepsilon \mathrm{d} V=\int_{V} \delta \mathbf{u}^{T} \mathbf{b} \mathrm{d} V+\int_{S} \delta \mathbf{u}^{T} \mathbf{t} \mathrm{d} S$,

$\int_{Y}\left[\delta \bar{\varepsilon} \bar{\varepsilon}+\bar{c}(\nabla \delta \bar{\varepsilon})^{T} \nabla \bar{\varepsilon}\right] \mathrm{d} V=\int_{V} \delta \bar{\delta} \bar{c} \mathrm{~d} V$

The finite element discretization is now rather straightforward. We interpolate the displacements $u$ and the 'non-local' equivalent strain $\bar{s}$ by

$\mathbf{u}=\mathbf{N a}$,

$\bar{\varepsilon}=\mathbf{h} \bar{\varepsilon}_{3}$

with $\mathrm{N}$ a matrix which contains the interpolation polynomials for the displacements and $h$ a row vector which contains the interpolation polynomials for the 'non-local' equivalent strains. The vectors a and $\bar{s}$ contain the nodal degrees of freedom for the displacements and the 'non-local' equivalent strains, respectively. The strains and the gradients of $\bar{\varepsilon}$ are then obtained as

$\varepsilon=\mathrm{Ba}$,

$\nabla \bar{\varepsilon}=\mathbf{Q} \bar{\varepsilon}$

where $\mathbf{B}=\mathbf{L N}$ and $Q=\nabla h$ contain the derivatives of the shape functions assembled in $\mathbf{N}$ and $\mathbf{h}$, respectively. Inserting identities (23)-(26) into Eqs. (21) -(22) yields

$$
\begin{gathered}
\int_{V} \delta \mathbf{a}^{T} \mathbf{N}^{T} \mathbf{R} \mathbf{N} \mathbf{a} \mathrm{d} V+\int_{V} \delta \mathbf{a}^{T}(1-\omega) \mathbf{B}^{T} \mathbf{D}^{\varepsilon} \mathbf{B a d} V \\
=\int_{V} \delta \mathbf{a}^{T} \mathbf{N}^{T} \mathbf{b} \mathrm{d} V+\int_{S} \delta \mathbf{a}^{T} \mathbf{N}^{T} \mathbf{t} \mathrm{d} S, \\
\int_{V} \delta \bar{\varepsilon}^{T}\left[\mathbf{h}^{T} \mathbf{h}+\bar{c} \mathbf{Q}^{T} \mathbf{Q}\right] \bar{\varepsilon} \mathrm{d} V=\int_{V} \delta \bar{\varepsilon}^{T} \mathbf{h}^{T} \tilde{z} \mathrm{~d} V .
\end{gathered}
$$

Note that the structure of Eqs. (27)-(28) is such that for the interpolants of the displacements as well as for the interpolants 
of the equivalent strain $\bar{c}$ we can use $C^{d}-$ continuous functions. However, to avoid stress oscillations the interpolation of the displacements should be one order higher than that of the equivalent strains $\bar{\varepsilon}$.

The set of Eqs. (27)-(28) is highly non-linear and must be solved using an iterative procedure at structural level. The Newton-Raphson procedure is widely used for this purpose and has also been adopted in this study. This algorithm requires the hinearization of the above set of equations. Care must be exercised that this linearization is carried out in a consistent fashion, since else the quadratic convergence of the method is lost (Simo and Taylor 1985). This linearization process has been carried out by Peerlings, de Borst, Brekelmans and de Vree (1995a, 1995b) for this gradient-enhanced damage relation and results in:

$\left[\begin{array}{ll}\mathbf{M}_{a a} & 0 \\ \mathbf{0} & 0\end{array}\right]\left[\begin{array}{l}\ddot{\mathbf{a}} \\ \ddot{\bar{\varepsilon}}\end{array}\right]+\left[\begin{array}{ll}\mathbf{K}_{a i a} & \mathbf{K}_{a b} \\ \mathbf{K}_{\varepsilon a} & \mathbf{K}_{\varepsilon \varepsilon}\end{array}\right]\left[\begin{array}{l}\mathrm{da} \\ \mathrm{d} \bar{\varepsilon}\end{array}\right]=\left[\begin{array}{l}\mathbf{f}_{e}+\mathbf{f}_{a} \\ \mathbf{f}_{\xi}-\mathbf{K}_{\varepsilon t} \bar{\varepsilon}_{j-1}\end{array}\right]$

where the d-symbol denotes the iterative improvement between two successive equilibrium iterations, $j$ is the iteration counter and

$$
\begin{aligned}
& \mathbf{f}_{e}=\int_{V} \mathbf{N}^{T} \mathbf{b d} V+\int_{S} \mathbf{N}^{T} \mathbf{t d} S, \\
& \mathbf{f}_{a}=-\int_{v} \mathbf{B}^{T} \boldsymbol{\sigma}_{j-1} \mathrm{~d} V, \\
& \mathbf{f}_{\varepsilon}=\int_{V} \mathbf{h}^{T \tilde{\sigma}_{j-1}} \mathrm{~d} V, \\
& \mathbf{M}_{a t}=\int_{V} \mathbf{N}^{\mathrm{T}} \mathbf{R N d V} \\
& \mathbf{K}_{a u}=\int_{V}\left(\mathbf{1}-\omega_{j-1}\right) \mathbf{B}^{T} \mathbf{D}^{c} \mathbf{B} \mathrm{d} V, \\
& \mathbf{K}_{\varepsilon \varepsilon}=\int_{V}\left(\mathbf{h}^{T '} \mathbf{h}+\bar{c} \mathbf{Q}^{\prime} \mathbf{Q}\right) \mathrm{d} V, \\
& \mathbf{K}_{\varepsilon a}=-\int_{V} \mathbf{h}^{T} \frac{\partial \tilde{\varepsilon}}{\partial r} \mathbf{B d} V, \\
& \mathbf{K}_{x s}=-\int_{\gamma} \frac{\partial \omega}{\partial \kappa} \frac{\partial k}{\partial \tilde{\varepsilon}} \mathbf{B}^{T} \mathbf{D}^{\varepsilon} \boldsymbol{\varepsilon}_{j-1} \mathbf{h d} V_{\nu}
\end{aligned}
$$

where $\lambda k_{c} / \partial \tilde{\varepsilon}$ equals 1 upon loading and wanishes for unloading. Note that the nonsymmetry in $\mathrm{Eq} .(29)$ is caused by the damage formalism, and not by the gradient enhancement. Only for special choices of the local equivalent strain $\widetilde{z}$ symmetry can be recovered (Simo and Ju 1987).

\section{2}

\section{Example}

To illustrate the performance of the numerical method, a simple, one-dimensional bar of length $L=100 \mathrm{~mm}$ is considered, Fig. 1. It is subjected to a uniaxial, pure tensile loading enforced by indirect displacement control of the end displacement $\bar{u}$ of the bar. While all material characteristics are uniform over the entire bar, in the centre of the bar a weakened zone with a length $d=10 \mathrm{~mm}$ and a $10 \%$ reduction in cross-sectional

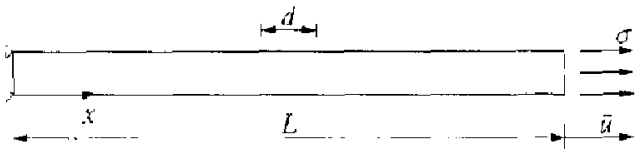

Fig 1. Imperfect bar in uniaxia! tension

area has been made in order to trigger localization of deformation in this area. For the case of "ideal-plasticity like damage', in which after a linear-elastic ascending branch, a constant stress at increasing damage is assumed, an analytical solution has been worked out by Peerlings, de Borst, Brekelmans and de Vree (1995b). The numerical solution appeared to agree exactly with this analytical solution.

Here, we shall consider a linear-softening type damage relation, Fig. 2, such that

$\omega(\kappa)=0$ if $\kappa \leqq \kappa_{0}, \quad \omega(\kappa)=\frac{\kappa_{u}\left(\kappa-\kappa_{0}\right)}{\kappa\left(\kappa_{t}-\kappa_{0}\right)}$ if $\kappa_{0} \leqq \kappa \leqq \kappa_{u}$

else $\omega(\kappa)=1$

with $\kappa_{0}=10^{-1}$ and $\kappa_{u}=0,0125$. Young $g^{2} \mathrm{~s}$ modulus has been set equal to $E=20,000 \mathrm{MPa}$ (so that for the tensile strength we have $\sigma_{1}=2 \mathrm{MPa}$ ) and as reference value for the gradient parameter $\bar{c}=1 \mathrm{~mm}^{3}$ has been adopted.

Numerical solutions has been obtained for successive levels of mesh refinement, namely with $80,160,320$ and 640 elements with a quadratic interpolation for the displacements and a linear interpolation for the 'nor-local' equivalent strain 5 . The results in terms of load-displacement curves are given in Fig. 3, which shows a cleat convergence towards a physically meaningful solution with a finite energy dissipation. Figure 4 shows the evolution of the damage and strain profiles for the finest mesh. We observe the formation of an initially relatively large damaged area, gradually evolving into a narrow zone of intense deformation and damage. This behaviour is fundamentally different from that when the same problem is analysed with a gradient plasticity theory. Then, a localization zone of constant thickness is predicted. However, the effect of the gradient parameter $\bar{c}$ on the structural response is similar for both enhanced continum models. A larger value of $\bar{c}$ leads to a more ductile response and a larger localization

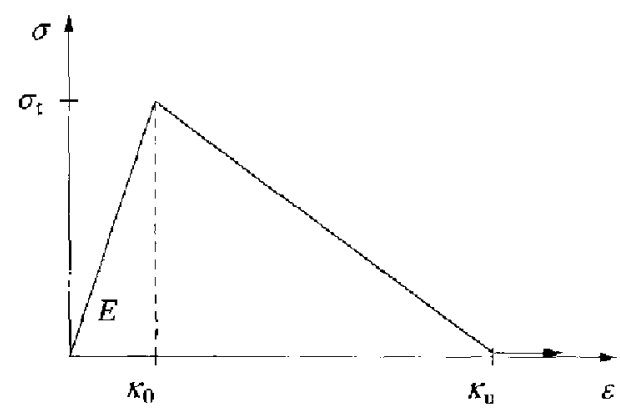

Fig 2. Linear (local) softening diagram 


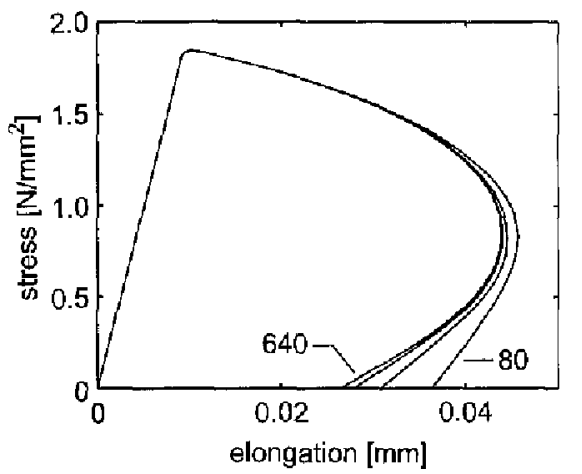

Fig. 3. Load-displacement curves for gradient daniage relation upon mesh refinement

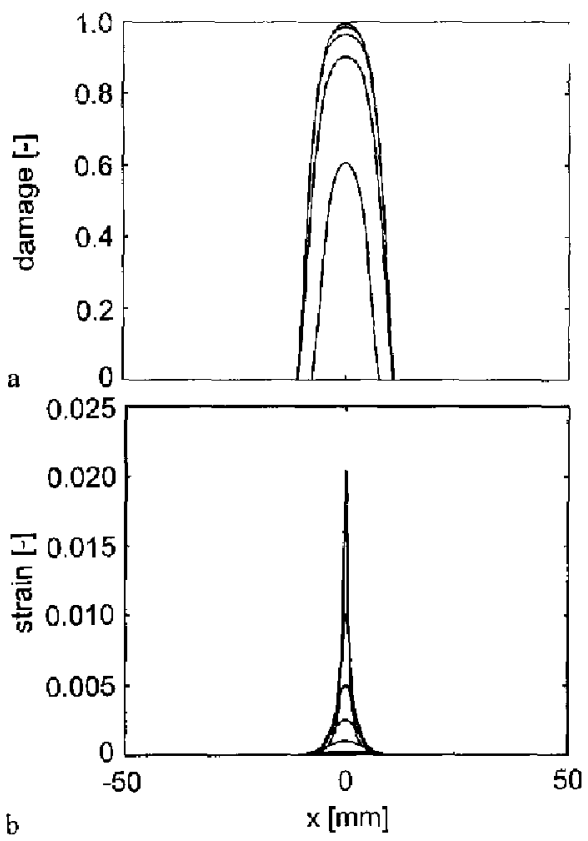

Fig. $4 \mathfrak{a}-\mathbf{b}$. Evolution of damage profiles (top) and strain profiles (bottom) along the bar

area, Fig. 5 , where the results of the calculation with the finest mesh are presented for $\bar{c}=0.25,1.00$, and $4.00 \mathrm{~mm}^{2}$. It is also observed that a higher peak load is found for increasing values of $\bar{c}$, which is due to the fact that the spreading of deformation delays the onset of the damage process.

\section{4}

\section{Algorithmic treatment of gradient plasticity}

\section{1}

\section{General}

Equation (14) shows the necessity to compute second-order gradients of the equivalent plastic strain measure $\kappa$. One possibility to achieve this is to use finite differences (Lasry and Belytschko 1988). The algorithm is then a secuence of separate solutions of the equilibrium problem using finite elements and the plastic yielding problem using finite differences. A more general approach (Mühlhaus and Aifantis 1991, de Rorst and Mïhlhaus 1992) is to use only finite elements
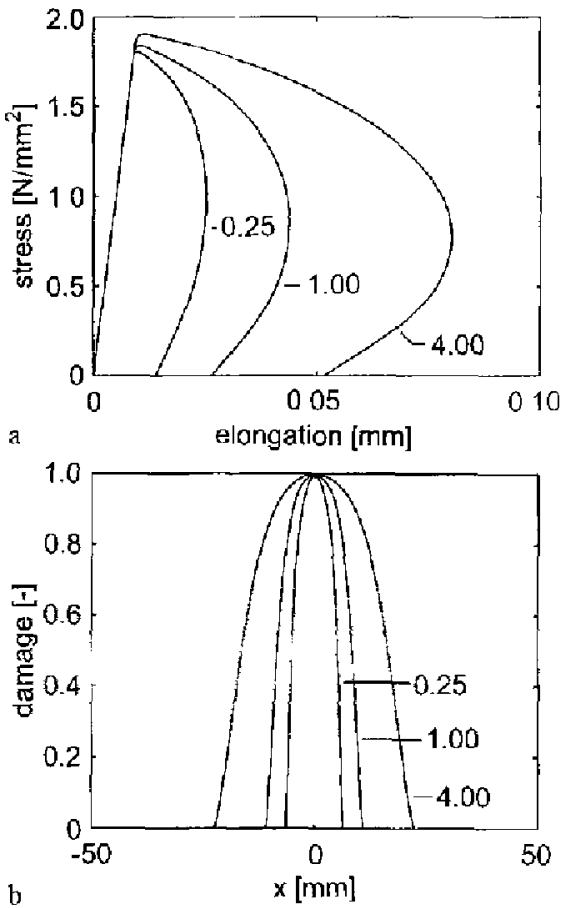

Fig. 5a-b. Influence of the internal length scale $\sqrt{\bar{c}}$ on the load-displacement curves (top) and the damage profiles (bottorn)

and to solve the two (coupled) problems simultaneously. For this purpose, it is necessary to employ a weak satisfaction of the yield condition and to discretize the plastic strain field in addition to the standard discretization of the displacement field, in a spirit similar to that used in the preceding section of gradient damage:

$\int_{V} \delta \mathbf{u}^{T}\left(\mathbf{L}^{T} \boldsymbol{\sigma}+\mathbf{b}-\mathbf{R} \ddot{\mathbf{u}}\right) \mathrm{d} V=\mathbf{0}$

and

$\int_{V} \delta \lambda f\left(\sigma_{j}, \kappa_{j}, V^{2} \kappa_{j}\right) d V_{l}=0$

where the subscript $j$ refers to the current iteration and $V_{2}$ denotes the volume of the plastic subdomain. Unlike the standard algorithms for plasticity, the yield condition is satisfied in a distributed sense. Furthermore, it is only fulfilled when convergence is reached and not necessarily during the iterative process.

Equation (39) can be modifed using integration by parts, the standard boundary conditions (5) 1 and decomposing $\sigma_{r}=\sigma_{j-k}+\mathrm{d} \sigma$ :

$\int_{Y} \delta \mathbf{u}^{T} \mathbf{R u ̈ d} V+\int_{V} \delta \varepsilon^{T} \mathrm{~d} \boldsymbol{\sigma} \mathrm{d} V$
$\quad=\int_{Y} \delta \mathbf{u}^{T} \mathbf{b} \mathrm{d} V+\int_{Y} \delta \mathbf{u}^{T} \mathrm{t} \mathrm{d} S-\int_{V} \delta \varepsilon^{T} \sigma_{f-1} \mathrm{~d} V$.

Furthermore, the yield function $f$ in Eq. (14) is developed in a Taylor series around $\left(\sigma_{j-1}, \kappa_{t-1}, \nabla^{2} \kappa_{f-1}\right)$ and truncated after the 
linear terms:

$f\left(\sigma_{j}, \kappa_{j}, \nabla^{2} \kappa_{j}\right)=f\left(\sigma_{j^{-1}}, \kappa_{j}, \nabla^{2} \kappa_{j \cdot 1}\right)+n^{T} \mathrm{~d} \sigma-h \mathrm{~d} \lambda+g \nabla^{2}(\mathrm{~d} \lambda)$,

where the following definitions have been used:

$\mathbf{n}=\frac{\partial f}{\partial \boldsymbol{\sigma}}$

$h=-\frac{\mathrm{d} \kappa}{\mathrm{d} \lambda} \frac{\partial f}{\partial \kappa}=-\eta \frac{\partial f}{\partial \kappa}$

and

$g=\frac{\mathrm{d} \kappa}{\mathrm{d} \lambda} \frac{\partial f}{\partial \nabla^{2} \kappa}=\eta \bar{c}$

Substitution of Eq. (42) into Eq. (40) then leads to;

$$
\begin{gathered}
\int_{V,} \delta \lambda\left[\mathrm{n}^{T} \mathrm{~d} \sigma-h \mathrm{~d} \lambda+g \nabla^{2}(\mathrm{~d} \lambda)\right] \mathrm{d} V_{i} \\
\quad=-\int_{V_{i}} \delta \lambda f\left(\sigma_{j-1}, K_{1-1}, \nabla^{2} K_{j-1}\right) \mathrm{d} V_{i}
\end{gathered}
$$

To proceed further, we must first integrate the differential stress-strain relation (13) for finite increments of strain. This stress update is done in a 'total-incremental' way, i.e. in every iteration the new estimate for the stress is computed starting from the equilibrium state $\sigma_{0}$ at the end of the previous loading step:

$$
\sigma_{j}=\sigma_{0}+\mathrm{D}^{2} \Delta \varepsilon_{j}-\Delta \lambda_{j} \mathrm{D}^{2} \mathrm{~m}_{\mathrm{j}}
$$

with the $\Delta$-symbol denoting the difference between a value in the current iteration $j$ and its value at the beginning of the loading step. Basically, Eq. (47) constitutes an Euler backward algorithm. The flow direction $m$ is approximated by $\mathbf{m}_{i}=\mathbf{m}\left(\sigma_{t}\right)$, its value in the trial stress state $\sigma_{t}=\sigma_{0}+D^{c} \Delta \varepsilon_{\mathrm{j}}$. The increment of plastic multiplier $\Delta \lambda$ is directly interpolated from the nodal values. For integration points in an elastic state an artificial hardening modulus $h=E$ is substituted to avoid singularity. The difference between the stress increments in two successive equilibrium iterations can be approximated by the linearization

$\mathrm{d} \sigma=\mathrm{D}^{\varepsilon} \mathrm{d} \varepsilon-\mathrm{d} \lambda \mathrm{D}^{s} \mathrm{~m}_{j-l}-\Delta \lambda_{y-1} \mathrm{D}^{\underline{e}} \frac{\hat{\gamma} \mathrm{m}_{\mathrm{y}-1}}{\partial \sigma_{j-1}} \mathrm{~d} \sigma$.

Upon introduction of the pseudo-elastic stiffness operator $\mathbf{H}$ :

$\mathbf{H}=\left[\left(\mathrm{D}^{e}\right)^{-1}+\Delta \lambda_{j-1} \frac{\partial \mathbf{m}_{\mathbf{j}-1}}{\partial \sigma_{j-1}}\right]^{-1}$,

this relation can be rearranged as

$\mathrm{d} \boldsymbol{\sigma}=\mathbf{H}\left(\mathrm{d} \varepsilon-\mathrm{d} \lambda \mathbf{m}_{1-\mathrm{t}}\right)$
We next substitute Eq. (50) into Eqs. (41) and (46), so that the following relations ensue:

$$
\begin{aligned}
& \int_{V} \delta \mathbf{u}^{T} \mathbf{R u ̈ d} V+\int_{V} \delta \boldsymbol{\varepsilon}^{T} \mathbf{H}(\mathrm{d} \boldsymbol{\varepsilon}-\mathrm{d} \lambda \mathbf{m}) \mathrm{d} V \\
& =\int_{V} \delta \mathbf{u}^{T} \mathbf{b} \mathrm{d} V+\int_{S} \delta \mathbf{u}^{T} \mathbf{t} \mathrm{d} S-\int_{V} \delta \varepsilon^{T} \boldsymbol{\sigma}_{y-1} \mathrm{~d} V
\end{aligned}
$$

and

$$
\begin{gathered}
\int_{V_{i}} \delta \lambda\left[\mathbf{n}^{T} \mathbf{H d} \varepsilon-\left(h+\mathbf{n}^{T} \mathbf{H m}\right) \mathrm{d} \lambda+g \nabla^{2}(\mathrm{~d} \lambda)\right] \mathrm{d} V_{2} \\
=-\int_{V_{j}} \delta \lambda f\left(\sigma_{j-1}, K_{j-1}, V^{2} k_{j-1}\right) \mathrm{d} V_{\eta}
\end{gathered}
$$

where the values of $\mathbf{n}, \mathbf{m}, h$ and $g$ on the left hand side of Eq. (52) are determined at the end of iteration $j-1$, i.e. for the state defined by $\left(\sigma_{j-1}, \kappa_{j-1}, \nabla^{2} \kappa_{j-1}\right)$.

Using integration by parts for the last term on the left hand side of $\mathrm{Eq}$. (52) we obtain

$$
\begin{gathered}
\int_{V_{i}} \delta \lambda\left[\mathbf{n}^{T} \mathrm{Hd} s-\left(h+\mathbf{n}^{T} \mathrm{Hm}\right) \mathrm{d} \lambda\right] \mathrm{d} V_{i}-\int_{V_{i}} g(\nabla \delta \lambda)^{T}(\nabla \mathrm{d} \lambda) \mathrm{d} V_{\lambda} \\
=-\int_{V_{i}} \delta \lambda f\left(\sigma_{j-1}, \kappa_{j-1}, \nabla^{2} \kappa_{j-1}\right) \mathrm{d} V_{\lambda}
\end{gathered}
$$

provided the non-standard boundary conditions

$\delta \lambda=0$ or $(\nabla d \lambda)^{T} v_{s}=0$

are fulfilled on the elasto-plastic boundary $S_{\lambda}$ of the body. The first condition is delicate for finite increments, since the elastic plastic boundary moves when the plastic zone in the body evolves. During this process the boundary condition $\delta \dot{\lambda}=0$ on the momentary elastic-plastic boundary may not be true and (54) must hold. If the same mesh is used for both the equilibrium and yield condition, i.e. if integrals over the plastic subdomain $v$; are replaced by integrats over the whole body $V$ in Eqs. (52) or (53), either the admissible 82 must vanish in the elastic part of the body, which would set constraints on the shape functions to be used, or we must enforce there $f=0, \mathbf{n}=\mathbf{m}=\mathbf{0}$ and $\mathbf{d} \lambda=0$. This is a major difference with gradient damage, where the Helmholt $z$ equation for the non-local strain variable must be satisfied over the whole domain throughout the entire loading process. It may partly be the explanation why a proper spread of the damage zone is observed in gradient damage when $C^{a}$-continuous interpolants are used for the averaged equivalent strain, while deformation is trapped into a single element when the same interpolation functions are used for gradient plasticity. Then, a proper spread can only be achieved if $C^{\prime}$-interpolation polynomials are used for the equivalent plastic strain, or if a penalty approach is used which, at the elastic-plastic boundary, enforces $(54)_{2}$.

\section{2}

\section{$C^{1}$-formulation}

In the field Eqs. (51) and (52)/(53) there appear at most first-order derivatives of the displacements. Therefore the discretization procedure for the displacement field $\mathbf{u}$ requires 
$C^{\mathbf{0}}$-continuous interpolation functions assembled in $\mathrm{N}$, similar to Eq. (23). The strains are then derived according to Eq. (25). However, as discussed in the previous section, the discretization of the plastic multiplier $\lambda$ requires $C^{\perp}$-continuous shape functions:

$\lambda=\mathbf{h} \mathbf{A}$

where $A$ is a vector with nodal degrees of freedom of the plastic multipliers. Introducing a matrix $Q$ which contains the derivatives of the shape functions in $h$ we obtain the discretization of the gradient of the plastic multiplier:

$\nabla \lambda=\mathrm{Q} A, \quad \mathrm{Q}=\nabla \mathrm{h}$

Introducing a row vector $\mathbf{p}$ which contains the Laplacians of the shape functions in $h$, we find the discretization formula for the Laplacian of the plastic multiplier:

$\nabla^{2} \lambda=p \Lambda$

After substitution of the above identities into Eqs. (51) and (52), and requiring that the result must hold for any admissible variation of $\delta$ and $\delta A$, we obtain the following set of algebraic equations:

$\left[\begin{array}{ll}\mathbf{M}_{a a} & \mathbf{0} \\ \mathbf{0} & \mathbf{0}\end{array}\right]\left[\begin{array}{l}\ddot{\mathbf{a}} \\ \ddot{\boldsymbol{X}}\end{array}\right]+\left[\begin{array}{ll}\mathbf{K}_{a a} & \mathbf{K}_{u j} \\ \mathbf{K}_{j a} & \mathbf{K}_{j, i}\end{array}\right]\left[\begin{array}{l}\mathbf{d a} \\ \mathbf{d} \mathbf{A}\end{array}\right]=\left[\begin{array}{c}\mathbf{f}_{z}+\mathbf{f}_{a} \\ \mathbf{f}_{j}\end{array}\right]$,

with the pscudo-elastic stiffness matrix:

$\mathrm{K}_{i a}=\int_{V} \mathbf{B}^{T} \mathbf{H B} \mathrm{d} V$

the off-diagonal matrices:

$\mathbf{K}_{u \lambda}=-\int_{V} \mathbf{B}^{\tau} \mathbf{H m h d} V$,

$\mathbf{K}_{2 \alpha}=-\int_{V} \mathbf{h}^{T} \mathbf{n}^{T} \mathbf{H B d} V$,

the nonsymmetric gradient-dependent matrix:

$\mathbf{K}_{z z}=\int_{V}\left[\left(h+\mathbf{n}^{T} \mathbf{H m}\right) \mathbf{h}^{\top} \mathbf{h}-g \mathbf{h}^{\top} \mathbf{p}\right] \mathrm{d} V$.

The mass matrix $\mathbf{M}_{a, a}$, the external force vector $\mathbf{f}_{a}$ and the vector of nodal forces caused by the internal stresses $\mathbf{f}_{a}$ are as in Eqs. (33), (30) and (31), and the vector of residual forces emerging from the inexact fulfilment of the yield condition reads:

$\mathbf{f}_{i}=\int_{V} \mathbf{h}^{T} f\left(\sigma_{j-1}, \kappa_{j-1}, \nabla^{2} \kappa_{j-1}\right) \mathrm{d} V$.

If we make use of Eq. (53) instead of Eq. (52), a symmetric form of the discretized yield condition is obtained, which leads to a similar matrix problem as in Eq. (58), but now the nonsymmetry due to the laplacian term has vanished, i.e. the matrix $\mathbf{K}$ can be nonsymmetric only because of non-associated flow $(\mathbf{m} \neq \mathbf{n})$ :

$\mathbf{K}_{\lambda}=\int_{V}\left[\left(h+\mathbf{n}^{T} \mathbf{D}^{e} \mathbf{m}\right) \mathbf{h}^{T} \mathbf{h}+g \mathbf{Q}^{T} \mathbf{Q}\right] \mathrm{d} V$.
However, the additional boundary conditions (54) have to be enforced in this case.

\section{3}

\section{$C^{0}$-formulation}

In order to be able to use $C^{2}$-continuous interpolation functions for the plastic multiplier field, we introduce new variables $\phi_{x}$, $\phi_{y}$ and $\phi_{z}$ :

$\phi_{x}=\frac{\partial \lambda}{\partial x}, \quad \phi_{y}=\frac{\partial \lambda}{\partial y}, \quad \phi_{z}=\frac{\partial \lambda}{\partial z}$

and collect them in a vector $\phi=\left(\phi_{x}, \phi_{y}, \phi_{z}\right)$. Accordingly, we can write the gradient of the plastic multiplier as

$\nabla \lambda=\phi$

and represent the Laplacian of $\lambda$ as

$\nabla^{2} \lambda=\nabla^{T} \phi$

where the scalar product of the operator $\nabla^{T}$ and the vector field $\phi$ denotes the divergence operator. The result of Eq. (67) can be substituted in Eq. (52) or (53), but the constraint (66) must be added to the formulation.

Since we want to avoid the introduction of a Lagrangian multiplier field in addition to the already defined three fields $\mathbf{u}$, $\lambda$ and $\phi$, we will make use of a penalty approach. We can include the constraint by means of an additional variational equation:

$\int_{V} k(\nabla \lambda-\phi)^{T}[\nabla(\delta \lambda)-\delta \phi] \mathrm{d} V=0$

where $k$ is a penalty factor. In computations we use $k=E^{3}$, where $E$ is Young's modulus. Using the incremental form of Eq. (68) together with Eqs. (51) and (52) we obtain a set of three integral equations:

$$
\begin{aligned}
& \int_{V} \delta \mathbf{u}^{T} \operatorname{Rü} \mathrm{d} V+\int_{V}^{t} \delta \varepsilon^{T} \mathrm{H}(\mathrm{d} \varepsilon-\mathrm{d} \lambda \mathrm{m}) \mathrm{d} V \\
& \quad=\int_{V} \delta \mathbf{u}^{T} \mathrm{~b} \mathrm{~d} V+\int_{S} \delta \mathbf{u}^{\mathrm{I}} \mathbf{t} \mathrm{d} S-\int_{V} \delta \varepsilon^{T} \sigma_{j-1} \mathrm{~d} V, \\
& \int_{V} \delta \lambda\left[\mathbf{n}^{T} \mathrm{Hd} \varepsilon-\left(h+\mathbf{n}^{T} \mathrm{Hm}\right) \mathrm{d} \lambda+g V^{T} \mathrm{~d} \phi\right] \mathrm{d} V \\
& \quad=-\int_{V} \delta \lambda f\left(\sigma_{1-1}, \kappa_{j-1}, V^{2} h_{j-1}\right) \mathrm{d} V,
\end{aligned}
$$

where according to Eqs. (18) and (67) we calculate $\nabla^{2} k=\eta \nabla^{r} \phi$ and

$$
k \int_{V} \delta \lambda \nabla^{T}[\nabla(\mathrm{d} \lambda)-\mathrm{d} \phi] \mathrm{d} V-k \int_{V} \delta \phi^{T}[\nabla(\mathrm{d} \lambda)-\mathrm{d} \phi] \mathrm{d} V=0 .
$$

The above equations are discretized using the formulas $\{23\}$, (25), (55) and (56), but now with $C^{\circ}$-continuous shape functions in $\mathbf{h}$, and the following interpolation for the new variables in $\phi$ :

$\phi=\mathbf{P} \boldsymbol{\phi}$ 
where $\boldsymbol{\phi}$ contains the nodal values of $\phi_{x}, \phi_{y}$ and $\phi_{x}$ and $\mathbf{P}$ is a matrix of shape functions, similar to $\mathrm{N}$. Upon the discretization of Eqs, (69)-(71) and the usual argument that the resulting equations mist hold for any admissible $\delta a, \delta \Lambda$ and $\delta \Phi$, we obtain the following set of algebraic equations in a matrix form:

$$
\left[\begin{array}{ccc}
\mathrm{M}_{a w} & 0 & 0 \\
0 & 0 & 0 \\
0 & 0 & 0
\end{array}\right]\left[\begin{array}{c}
a \\
\ddot{A} \\
\ddot{\Phi}
\end{array}\right]
$$

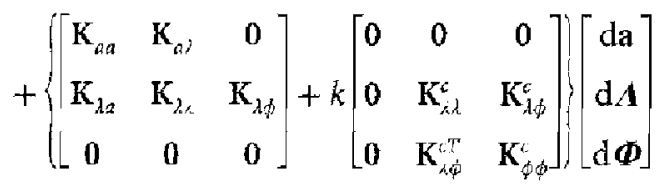

$$
\begin{aligned}
& =\left[\begin{array}{c}
f_{e}+f_{a} \\
f_{j}
\end{array}\right] .
\end{aligned}
$$

In Eq. (73) the matrices $M_{n a}, K_{n a}, K_{a,}$ and $K_{, a}$ are given in Eqs. (33), (59) $-(61)$. $K_{i \alpha}$ and $K_{\lambda \phi}$ are defined as

$\mathbf{K}_{\lambda \lambda}=\int_{\gamma^{T}}\left(h+\mathbf{n}^{T} \mathbf{H m}\right) \mathbf{h}^{T} \mathbf{h} \mathrm{d} V, \quad \mathbf{K}_{\lambda \phi}=-\int_{\gamma} g \mathbf{h}^{T} \nabla^{T} \mathbf{P} \mathrm{d} V^{\gamma}$

and the submatrices with the supersript $c$ in the additional (symmetric) matrix introducing the constraint (66) are defined as

$\mathbf{K}_{12}^{c}=\int_{y} \mathbf{Q}^{T} \mathbf{Q d} V, \quad \mathbf{K}_{\hat{\psi} \phi}^{c}=\int_{V} \mathbf{P}^{T} \mathbf{P} \mathrm{d} V, \quad \mathbf{K}_{x \phi}^{c}=-\int_{\mathrm{V}} \mathbf{Q}^{T} \mathbf{P} \mathrm{d} V$.

In this formulation all the interpolation functions in $\mathrm{N}, \mathrm{h}$ and $P$ are $C^{0}$-continuous.

If we substitute the new variables assembled in $\phi$ into Eq. (53), which is the starting point for the symmetric formulation, we obtain the weak form of the yield condition in which the derivatives of $\phi$ appear only on the right hand side. Substitution of the discrelization formulas (23), (25), (55) and (56) then gives

$$
\left[\begin{array}{ccc}
M_{a \mathfrak{a}} & 0 & 0 \\
0 & 0 & 0 \\
0 & 0 & 0
\end{array}\right]\left[\begin{array}{c}
\ddot{a} \\
\ddot{A} \\
\ddot{\phi}
\end{array}\right]
$$

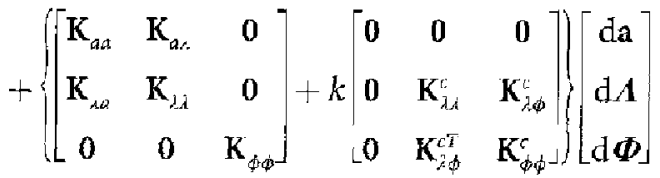

$$
\begin{aligned}
& =\left[\begin{array}{c}
\mathbf{f}_{\varepsilon}+\mathbf{f}_{\mathfrak{a}} \\
\mathbf{f}_{\mathfrak{z}} \\
0
\end{array}\right]
\end{aligned}
$$

where

$\mathbf{K}_{\phi \phi}=\int_{V} g \mathbf{P}^{\mathrm{T}} \mathbf{P d} V$

and all the other submatrices have been defined previously. With the set (76) the additional boundary conditions (54), are now written as:

$\delta \lambda=0$ or $\mathrm{d} \phi^{T} \boldsymbol{\nu}_{\lambda}=0$

For the penalty method to be successful the penalty submatrix $K^{c}$ must be singular. Otherwise non-zero $\boldsymbol{\Phi}$ values are not admitted. To achieve this goal reduced numerical integration should be used. Since the penalty constraint assures the satisfaction of Eq. (66) only in the sampling points, the best results are expected when uniformly reduced integration is employed for all the matrices.

\section{4}

\section{One-dimensional elements}

In the one-dimensional gradient plasticity elements with $C^{1}$-continuity the axjal displacement $u$ is interpolated linearly (denoted as $L 6 G$ ) or quadratically (denoted as $L 7 G$ ), and cubic Hermitian shape functions are used for the plastic multiplier $\lambda$. For element $L 6 G$ two-point Gaussian integration is employed. The submatrix $\mathbf{K}_{\lambda,}$ includes polynomials of the $6^{\text {th }}$-order and is not integrated accurately. Translation in the $x$-direction must be prevented and two additional constraints (e.g. symmetry) or boundary conditions for the $\lambda$-field must be introduced in the model. The element yields an exact fulfilment of the yield condition at the integration points, which means that when $\mathrm{f}_{\lambda} \rightarrow 0$, then $f \rightarrow 0$, but stress

oscillations are observed. This phenomenon may cause a failuxe of convergence in an early stage of the loading process (Panin 1994).

For element $L 7 G$ with two-point integration the balance between the interpolation for $u$ and $\lambda$ is optimal, i.e. the stress integration in Eq. (47) gives a stress state $\sigma$, which is constant within an element and which exactly fulfils the yield condition. Convergence in one iteration is observed unless the softening zone spreads or nonlinear softening is used (Pamin 1994). This behaviour is attributed to the special qualities of the integration stations, so-called Barlow points, in which higher-order accuracy of interpolated field derivatives is obtained.

The above properties are exhibited by the nonsymmetric formulation with $\mathrm{K}_{2,}$ from $\mathrm{Eq}$. (62). If the symmetric format for $K_{i,}$ according to $\mathrm{Eq} .(64)$ is used together with the required boundary conditions and two-point (reduced) integration, convergence is lost. This behaviour is attributed to an unfavourable numerical integration error, since for three-point integration the symmetric and nonsymmetric formulation give the same results. However, for the three-point integration too many constraints are introduced and the results are inaccurate. The stresses at one or more points are then mapped to the inside of the yield surface $(f<0)$, which violates the Kuhn-Tucker conditions and results in a disturbance of convergence.

Similarly, two different one-dimensional $C$-elements can be defined with separate Lagrange interpolation of the 
longitudinal displacement $u$, the plastic multiplier $\lambda$ and the additional variable $\phi$. Element $L 6 C$ uses linear shape functions and one integration point. It is the point, in which the constraint $\phi=\lambda_{, x}$ is fulfilled. The longitudinal translation must be prevented and two boundary conditions for $\lambda$ or $\phi$ should be introduced in a model. The element is perfectly convergent since the integration station is a Barlow point. Element $L 9 C$ uses quadratic shape functions and two Gauss points, which are again optimal for convergence. Boundary conditions similar to the $L 6 C$ element must be introduced and the return mapping is also exact. In presence of the additional boundary conditions (78) the symmetric and nonsymmetric formulations give the same results for the one-dimensional $\mathrm{C}^{0}$-elements, because the employed numerical integration schemes are sufficient for an exact integration of the shape function polynomials.

\section{5}

\section{Example}

As example problem, we shall again employ the imperfect bar in tension, Fig. 1. For this one-dimensional problem the following simple yield function holds:

$f=\sigma-\bar{\sigma}(\kappa)+\bar{c} \frac{\mathrm{d}^{2} \kappa}{\mathrm{d} x^{2}}$

with $\sigma$ the axial stress. The hardening parameter $\kappa$ is now equal to the plastic multiplier $\lambda$ and the axial plastic strain $2^{p}$. According to the analytical solution (de Borst and Muhlhaus 1992) the width of the localization zone $w$ is now constant and given by:

$w=2 \pi l$

with $t$ the internal length scale defined as

$I=\sqrt{-\frac{\bar{c}}{h}}$.

In Eq. (81) $h=\mathrm{d} \bar{\sigma} / \mathrm{d} \varepsilon^{2}$ is the hardening/softening modulus.

The same geometric data have been used as in the simulation with the gradient damage model. Also the Young's modulus and the tensile strength have been assigned the same value.The geometric imperfection has been replaced by a material imperfection such that the elements in the centre of the bar have a $10 \%$ reduction in tensile strength, namely $\sigma_{t}=$ $1.8 \mathrm{MPa}$ instead of $\sigma_{\mathrm{z}}=2.0 \mathrm{MPa}$. Linear softening has again been used with a softening modulus $h=-0.1 \mathrm{E}$. A value for the internal length scale $l=5 \mathrm{~mm}$ has been adopted, resulting in $\bar{c}=50,000 \mathrm{~N}$. The corresponding width of the localization zone is $w=31.4 \mathrm{~mm}$.

We begin the comparison with $C^{a}$-continuous penalty-enhanced elements. The left diagram of Fig. 6 shows load-displacement paths obtained using elements $L 6 C$ and $L 9 C$ with one-point and two-point Gauss integration, respectively. Immediate convergence has been observed in the calculations. While the coarse mesh with 20 linear $L 6 C$ elements still gives a slightly too stiff response and a disturbed $\lambda$ distribution, the fine mesh and both meshes for the quadratic $L 9 C$ element
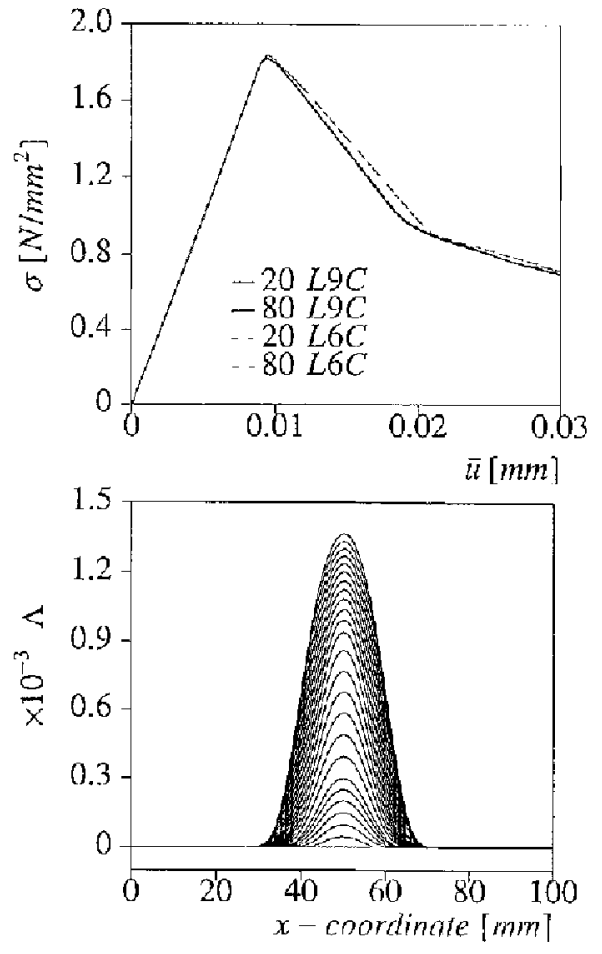

Fig. 6. Load-displacement diagrams (top) and evolution of the plastic strains (bottom) for $\mathrm{C}^{0}$ one-dimensional elements

yield identical results. When all the inelastic points are in the softening regime, the slope of the load-displacement diagram is equal to the analytical value. The calculations are also stable when the strain in the centre elements exits the softening branch $\left(\kappa>\kappa_{i t}\right)$. The load-displacement diagrams then bend upwards and the localization zone broadens. This behaviour is a result of the fact that, when the softening modulus $h$ goes to zero, while $\bar{c}$ is kept constant, the internal length 1 locally increases of infinity.

Next, we apply the $C^{1}$-continuous elements $L 7 G$ with quadratic interpolation of the displacement and Hermitian interpolation of the plastic multiplier. Two different values of the internal length $l$ are assumed: $l=5 \mathrm{~mm}$ (so that $\bar{c}=50,000 \mathrm{~N}$ and $w=31.4 \mathrm{~mm})$ and $l=2.5 \mathrm{~mm}(\bar{c}=12,500 \mathrm{~N}$ and $w=15.7 \mathrm{~mm}$ ). Figure 7 shows the load-displacement diagrams for these cases. As long as all the points in the structure remain in the softening regime, the results for the two meshes with 20 and 80 elements are practically the same, and equal to those for the $C^{0}$-elements. When in the centre points the linear softening branch is exited, the calculations only remain stable if a mesh is employed that is dense enough compared to the width of the localization zone (and therefore relative to the value of the gradient constant $\bar{c}$ ). Fig. 7 shows that the resuits for both meshes are similar for the larger regularization coefficient $\bar{c}=50,000 \mathrm{~N}$, but for the coarse mesh simulation of the case with $\vec{c}=12,500 \mathrm{~N}$ oscillations are observed.

The comparison of the wo diagrams in Fig. 8 shows that, similar to gradient damage, the internal length 7 (or alternatively, the gradient parameter $\bar{c}$ ) controls the width of localization zone in the linear softening regime. 


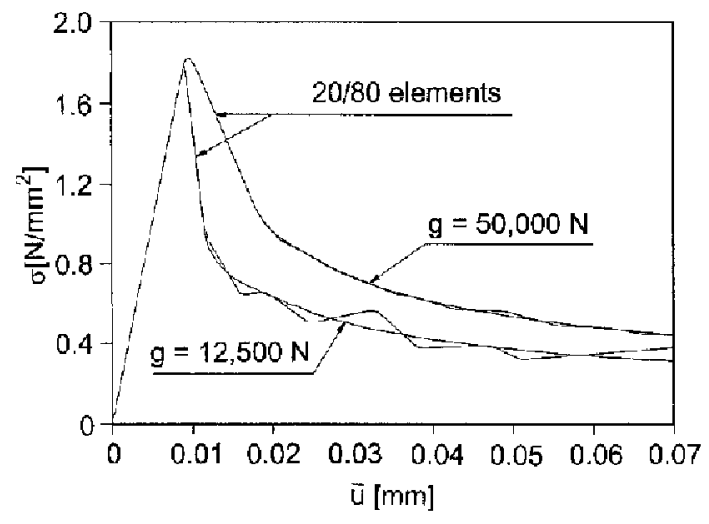

Fig. 7. Mesh sensitivity of load-displacement diagrams for the $L 7 G$ element
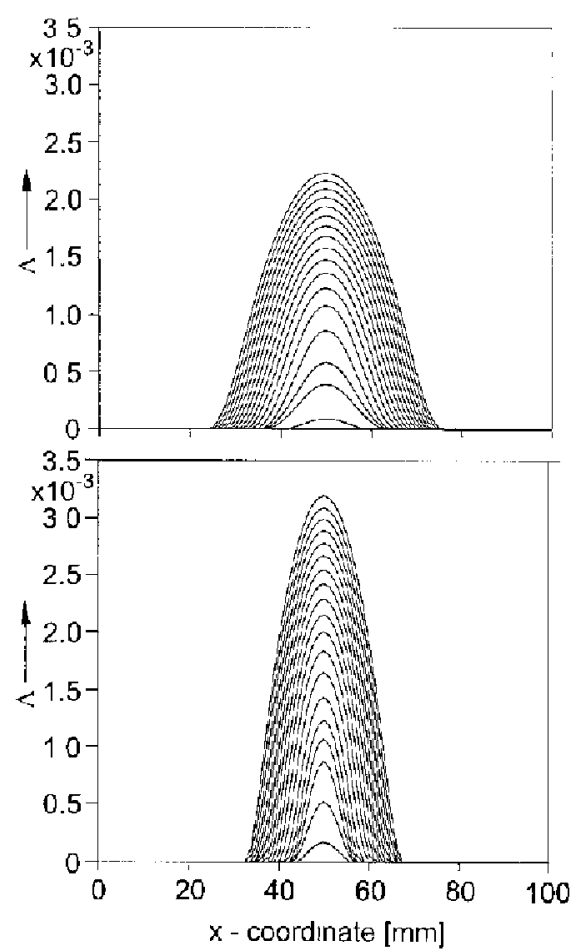

Fig. 8. Evolution of the plastic strains for different values of the gradient parameter

\section{5}

\section{Wave propagation and dispersion in gradient continua}

We conclude this contribution with a discussion on wave propagation and dispersion in gradient plasticity at the hand of the one-dimensional bar of Fig. 1 , now loaded by an impact load. In this dynamic context the governing equations for molion and continuity read

$\frac{\partial \sigma}{\partial x}=\rho \frac{\partial^{2} u}{\partial t^{2}}$

and

$\varepsilon=\frac{\partial u}{\partial x}$ with $u$ the axial displacement, $\sigma$ and $\varepsilon$ stress and strain and $x$ and $t$ spatial and temporal variables, respectively. With the strain decomposition

$\varepsilon=\varepsilon^{\varepsilon}+\varepsilon^{p}$,

and the bijective relation between stress $\sigma$ and elastic strain $\varepsilon^{2}$

$\sigma=E \varepsilon^{i}$,

the wave equation for one-dimensional gradient-dependent softening plasticity is derived as

$\frac{\bar{c}}{h+E}\left(E \frac{\partial^{4} u}{\partial x^{4}}-\rho \frac{\hat{\partial}^{4} u}{\partial x^{2} \partial t^{2}}\right)+\rho \frac{\partial^{2} u}{\partial t^{2}}-\frac{h E}{h+E} \frac{\partial^{2} u}{\partial x^{2}}=0$

For a dispersion analysis we consider a single harmonic wave which propagates through a one-dimensional element

$u(x, t)=A e^{(k x-i j t)}$.

The frequency $\omega$ is a function of the wave number $k$

$\omega=f(k)$,

and the function $f(k)$ is determined by the particular system under consideration. A system is considered to be dispersive if (Whitham 1974):

$f^{\prime \prime}(k) \neq 0$,

in which a prime denotes differentiation with respect to $k$. In this case, the phase speed

$c=\frac{Q}{k}$

is not the same for every wave number $k$ and modes represented by its wave number travel at different speeds and will therefore disperse. Finally, we adopt the standard definitions for the wave length

$\lambda=\frac{2 \pi}{k}$

and the period

$T=\frac{2 \pi}{\omega}$.

We substitute the harmonic wave solution (87) into Eq. (86). The dispersion relation for the gradient-dependent: softening plasticity system is then elaborated as

$\omega=c_{e} k \sqrt{\frac{h+\bar{c} k^{3}}{E+h+c k^{2}}}$ 
with $c_{e}=\sqrt{E / \rho}$ the so-called bar wave velocity. Now, the frequency is a real function of wave number $k$ if

$k \geq \sqrt{-\frac{h}{c}}$

or using Eq. (91)

$i \leqq 2 \pi l$,

with $l$ the internal length scale defined in Eq. (81). Equation

(94) states that there exists a cut-off value for $k$. This value of $k$ corresponds to the mode with the largest wave length that the gradient-dependent softening system can transmit. Above this value for $k$ all frequencies are real.

Equation (93) shows that for a non-zero gradient constant $\bar{c}_{3} f^{\prime \prime}(k) \neq 0$, so that wave propagation in the gradientdependent plasticity system is dispersive. The dispersion curves have been plotted in Fig. 9 for a linear-elastic system $\left.(\omega)=c_{e} k\right)$, a standard softening plasticity system (with imaginary frequencies $\omega=i c_{\varepsilon} k \sqrt{-h /(E+h))}$, and the gradient-dependent softening plasticity system. In the right part of Fig. 9 the corresponding phase velocity-wave number $(c-k)$ curve is shown. In Fig. 9 the bar wave velocity $c_{e}=1000 \mathrm{~m} / \mathrm{s}$, the Young's modulus $E=20,000 \mathrm{MPa}$, the softening modulus $h=-0.1 E$ and the gradient constant $\bar{c}=50,000 \mathrm{~N}$.

Dispersion in a softening system is closely related to the problem of localization of deformation. As a result of softening localization of deformation may occur and the behaviour of localized zones is very much dependent on the dispersive characteristics of the material. For a standard softening plasticity system the inability of the material to transmit waves with a real frequency (and phase velocity) causes the strains
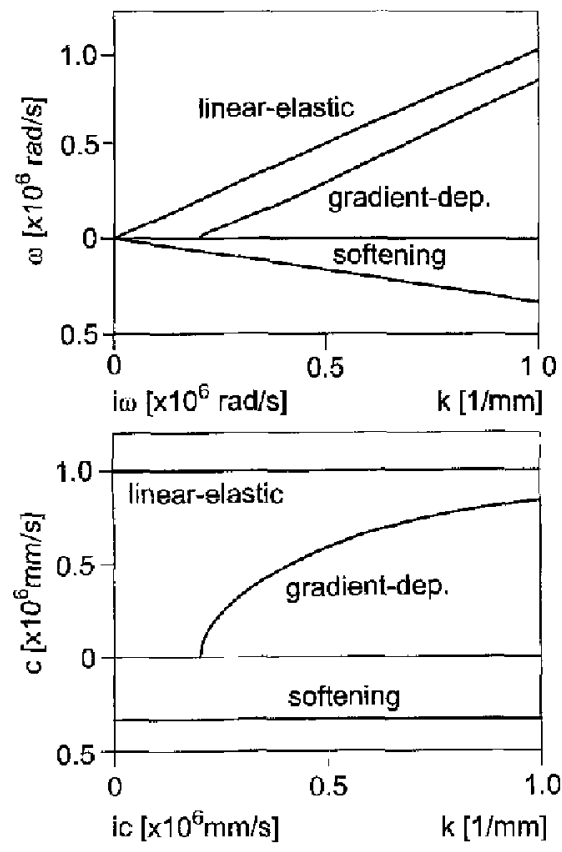

Fig. 9. Frequency (top) and phase velocity (bottom) as a function of the wave number to localize in zones of zero thickness. However the dispersion contribution due to spatial discretization causes the frequency to reach a real zero value, and numerically a stationary wave with frequency and phase velocity equal to zero is computed in the localization zone (Sluys, Cauvern and de Borst 1995). The solution is now mesh dependent and corresponds to this stationary wave. Its wave length $\lambda$ is equal to the width of the zone, namely a one finite element wide zone (for constant strain elements).

For the gradient-dependent softening system the fact that waves with real phase velocities disperse has the advantageous consequence that the localizalion zone can extend and that the strain profile in the localization zone can be transformed because different modes travel at different speeds. These features are of pivotal importance for simulating zones of localized deformation with a finite size instead of the zero-thickness solution as obtained for the standard softening plasticity system. Also for the gradient model the localization zone acts as a stationary wave with frequency and phase velocity equal to zero. For this reason the width of the localization zone $w$ is equal to the lowest-order wave that the gradient-dependent softening system can transmit, i.e. $w=2 \pi i$. The width of the localization zone apppears as a consequence of the length scale effect and the spurious mesh dependence is removed (Sluys, de Borst and Mühlhaus 1993).

In finite element formulations a discretization is carried out with respect to space and time. Discretization is another source of dispersion (Huerta and Pijaudier-Cabot 1994, Sluys and de Borst 1994) and is introduced irrespective of the fact whether the underlying material exhibits dispersion of waves (gradient-dependent softening system) or not (standard softening system). The dispersion contribution of temporal and spatial discretization has been treated in detail by Sluys, Cauvern and de Borst (1995) for a one-dimensional elasto-plastic bar with and without gradient influence.

\section{References}

Aifantis, E. C. 1984: On the microstructural origin of certain inelastic models. J. Fng. Mater. Technol. 106: 326-334

Aifantis, E. C. 1987; The physics of plastic deformation. Int. J. Plasticity 3: $211-247$

Aifantis, E. C. 1992: On the tole of gradients in the localization of deformation and fracture. Int. J. Eng. Sci. 30: 1279-1299

Bazant. Z. P.; Pijaudier-Cabot. G. 1988: Nonlocal continuum damage, localization instability and convergence. J. Appl. Mech. 55: 287-293 Borst, R. de 1991: Simulation of strain localisation: A reappraisal of the Cosserat continuum. Eng. Comp. 8: 317-332

Borst, R. de; Mühlhaus. H.-B. 1992: Gradient-dependent plasticity: Formulation and algorithmic aspects. Int. J. Num. Meth. Eng. 35: $521-539$

Borst, R. de 1993: A generalisation for $J_{2}$-flow theory for polar continua. Comp. Meth. Appl. Mech. Eng. 103: 347-362

Borst, R. de; Pamin, J.; Sluys, L. J. 1995: Gradient plasticity for localization problems in quasibrittle and frictional materials. In: Owen, D. R. J.; Onate, E.; Hinton, E. (eds): Proc. Fourth Int. Conf. on Computational Plastjcily, Theory and Applications, pp. 509-533. Swansea: Pineridge Press

Coleman, B. D.; Hodgdon, M. L. 1985: On shear bands in ductile materials. Arch. Ration. Mech. Anal, 90: $219-247$

Huerta, A.; Pijaudier-Cabot, G. 1994: Discretization influence on the regularization by two localization limiters. ASCE J. Eng. Mech. 120: $1198-1218$

Lasry, D.; Belytschko, T. 1988: Localization limiters in transient problems. Int. J. Solids Structures 24: 581-597 
Lemaitre, J.; Chaboche J. I. 1990: Mechanics of solid materials. Cambridge: Cambtidge University Press

Loret, B.; Prévost, J. H. 1990: Dynamic strain localization in elasto-(visco-) Plastic solids, Part 1. Comp. Meth. Appl. Mech. Eng. 83: $247-273$

Mühlhaus, H.-B.; Vardoulakis, I. 1987: The thickness of shear bands in granular materials. Geotechnique 37: 271 283

Mühlhaus, H.-B. 1989: Application of Cosserat theory in numerical solutions of limit load problems. Ing.-Arch. 59: 124-137

Mühlhaus, H.-B.; Aifantis, E. C. 1991: A variational principle for gradient plasticity, Int. J. Solids Structures 28: $845-858$

Needleman, A. 1988: Material rate dependence and mesh sensitivity in localization problems. Comp. Meth. Appl. Mech. Eng. 67: 69-86 Pamin, J, 1994: Gradient-dependent plasticity in numerical simulation of localization phenomena. Diss., Delft University of 'Technology, Delft Peerlings, R. H. J.; Borst, R. de; Brekelmans, W. A. M.; Vree, J. H. P. de 1995a: Computational modelling of gradient-enhanced damage for fracture and fatigue problems. In: Owen, D. R. J.; Onate, E.; Hinton, E. (eds): Proc. Fourth Int. Conf. on Computational Plasticity, Theory and Applications, pp. 975-986. Swansea: Pineridge Press

Peerlings, R. H. J.; Borst, R. de; Brekelmans, W. A. M., Vree, J. H. P. de 1995b: Gradient-enhanced damage for quasi-brittle materials. Int. J. Num. Meth. Eng, submitted for publication

Pijaudier-Cabot, G; Bazant, Z. P. 1987: Nonlocal damage theory. $\Lambda$ SCE J. Eng. Mech. 113: 1512-1533

Schrever, H. L.; Chen. Z. 1986: One-dimensional softening with

localization. ASME J. Appl. Mech. 53: 791-797
Simo, I. C.; Taylor, R. L. 1985: Consistent tangent operators for rate-independent elasto plasticity. Comp. Meth. Appl. Mech. Eng. 48: $101-118$

Simo, J. C.; Ju, J. W, 1987; Strain and stress-based continuum damage models: I. Formulation. Int. J. Solids Structures 23: 821-840

Simo, J. C. 1988: Strain softening and dissipation: a unification of approaches. In Mazars, J.; I Bazant, Z. P. (eds): Cracking and Damage: Strain Localization and Size Effect, pp. 440-461. London-New York: Elsevier

Sluys, L. I. 1992: Wave propagation, localisation and dispersion in sottening solids. Dissertation, Delft University of Technology, Delft. Sluys, L. J.; Borst, R. de 1992: Wave propagation and localisation in a rate-dependent cracked medium-Model formulation and one-dimensional examples. Int. J. Solids Structures 29: 2945-2958

Sluys, L. J.; Borst, R. de; Mühlhaus, H.-B. 1993: Wave propagation, localization and dispersion in a gradient-dependent medium. Int. J. Solids Structures 30: 1153-1171

Sluys, L. J.; Borst, R. de 1994: Dispersive properties of gradient-dependent and rate-dependent media. Mech. Mater. 18: $131-149$

Sluys, L. J.; Cauvern, M.; Borst, R. de 1995; Discretization influence in strain-softening problems. Eng. Comput. 12: $209-228$

Vardoulakis, I.; Aifantis, E. C. 1991: A gradient flow theory of plasticity for granular materials. Acta Mechanica 87: 197-217

Whitham, G. B. 1974: Linear and nonlinear waves. London and New York: Wiley 